\title{
Research on the IR Lock-in Thermography Based on the Image Sequence Processing for NDT
}

\author{
Liu Junyan', Wang Yang', Dai Jingmin ${ }^{3}$, Liu Hui ${ }^{4}$, Wang Zijun
}

${ }_{3 ., 5}^{1,24}$ School of Mechatronics Engineering, Harbin Institute of Technology, Harbin, P.R.China

${ }^{3,5}$ School of Electronic Engineering and Automation, Harbin Institute of technology, Harbin, P.R.China

Abstract: Optically excited Lock-in thermography (OLT) which is an active thermography technique for NDT is based on propagation and reflection of thermal waves which are launched from the surface into the inspected component by absorption of modulated radiation. Phase angle images obtained by superposition of the initial thermal wave and its internal reflection display hidden thermal structures down to a certain depth below surface. Defects are found by comparing the observed features with expected features provided by theory or by an intact reference sample. This technique has been widely applied to detect the defection of composite materials and structure in aerospace and automotive industry.

In this study, the law of sine modulated lamp is used for active heat source into sample, and the thermal wave image sequences are collected with JADE MWIR 550 FPA infrared camera made in France Cedip Company. The special image sequence file formation is studied and analyzed. The digital filter algorithm and thermal wave signal single sequence Fourier transform algorithm are researched to obtain the phase angle image of thermal wave on the surface of sample. The program of IR Lock-in thermography is developed by Visual C++ development platform. The experimental results show that the lock-in thermography software system is reached to the Cedip IR lock-in thermography technique advance level.

Key words: Thermal waves, Non-destructive test, Lock-in thermography, Fourier transform

Corresponding Author: Liu Junyan Ph.D, School of Mechatronics Engineering, Harbin Institute of Technology, 422 Postbox, Harbin, Post Zip: 150001, P.R.China, Tel: $86+451+86414557$ or 86+451+86413257 Email: LJYWLJ@hit.edu.cn or LJYWLJHIT@yahoo.com.cn

Appends:

1. The Fig. 1 shows the structure of standard defects sample.
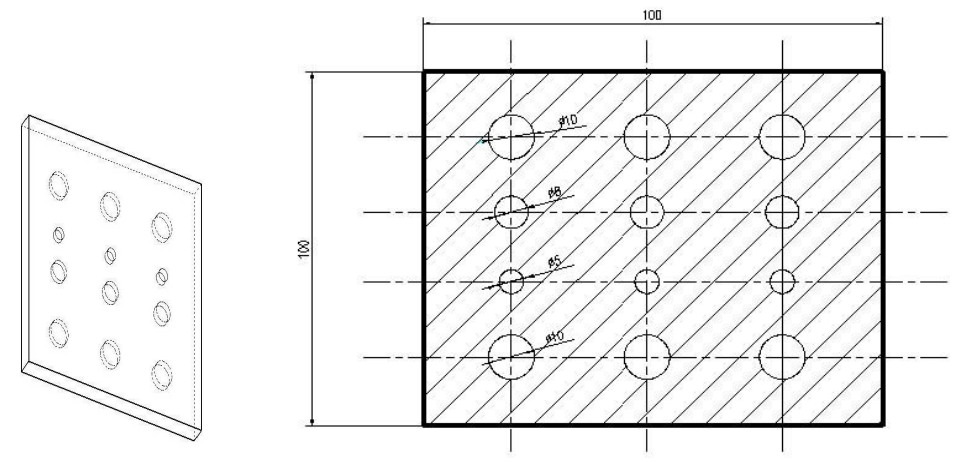

Fig. 1. The structure of sample

2. The Fig.2 shows the Cedip Lock-in thermography system for NDT.
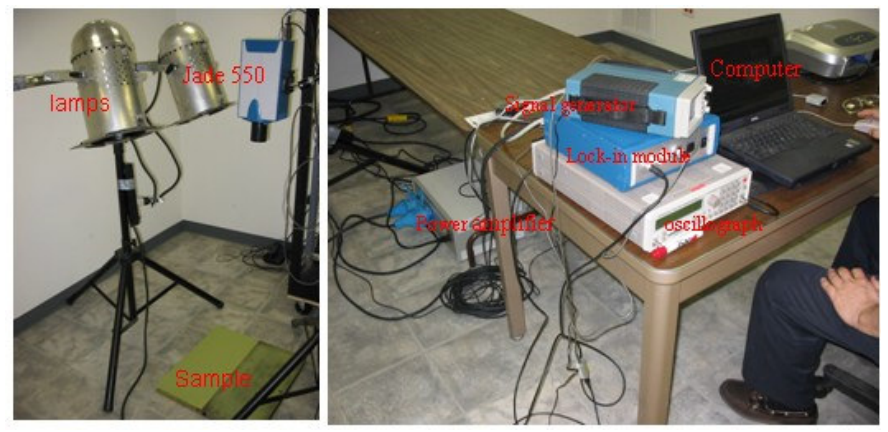

Fig. 2. Cedip JADE MWIR 550 Lock-in thermography system

3. The Fig.3 shows the software flow chart of the Lock-in thermography based on the image sequence processing 


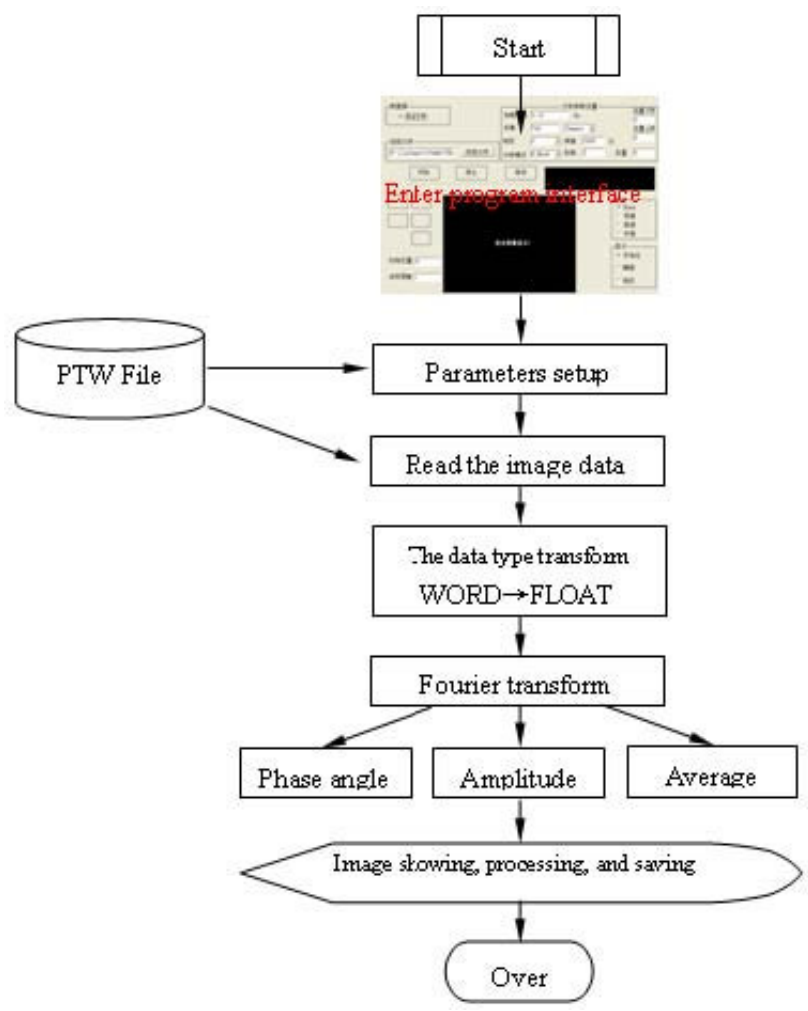

Fig. 3. The flow chart of the Lock-in thermography based on the image sequence processing

4. Results
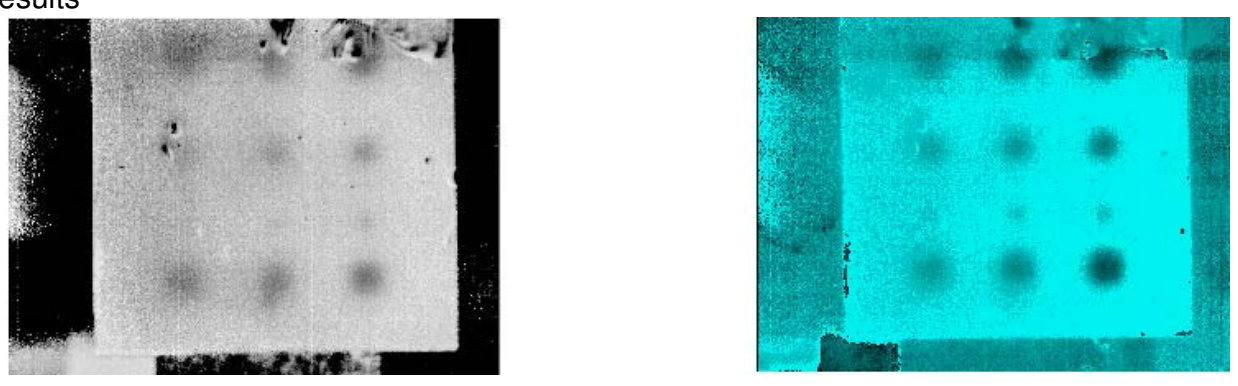

a) The phase angle image with Cedip Lock-b) The phase angle image with developed Lock-in in thermography system processing thermography software system processing

Fig.4 The comparison of phase angle image obtained 